\begin{tabular}{c} 
International Journal of Scientific World, $6(1)(2018) 19-26$ \\
International Journal of Scientific World \\
SPC \\
Website: $\begin{array}{c}\text { www.sciencepubco.com/index.php/IJSW } \\
\text { doi: } 10.14419 / i j s w . v 6 i 1.8662 \\
\text { Research paper }\end{array}$ \\
\hline
\end{tabular}

\title{
Bound lengths based on constant-stress PALT under different censoring patterns
}

\author{
Gyan Prakash $^{1 *}$ and Prabhakar Singh ${ }^{2}$ \\ ${ }^{1}$ Department of Community Medicine, M. L. N. Medical College, Allahabad, U. P., India \\ ${ }^{2}$ Department of Statistics, Harishchandra P. G. College, Varanasi, U. P., India \\ *Corresponding author E-mail:ggyanji@yahoo.com
}

\begin{abstract}
The Gompertz distribution is assumed in the present article for drawing the inferences based on Bayesian methodology. Constant-Stress Partially Accelerated Life Test (CS-PALT) have used for the underlying distribution on first-failure Progressive (FFP) censoring scheme. All special cases of the FFP censoring scheme have used for the present comparative analysis. The comparison has been done between different special cases of FFP based on Approximate Confidence Lengths (ACL) under Normal approximation, Bootstrap Confidence Length (BCL) and One-Sample Bayes Prediction Bound Lengths (BPBL). A simulation study have been carried out for the present analysis.
\end{abstract}

Keywords: Approximate Confidence Lengths (ACL), Bayes Prediction Bound Lengths (BPBL), Bootstrap Confidence Length (BCL), Constant-Stress Partially Accelerated Life Test (CS-PALT), First-Failure Progressive (FFP) Censoring Pattern.

\section{Introduction}

The Gompertz probability distribution now days get some more attention among researchers in different areas of technology, biological science, medical and natural sciences. The cumulative density function with scale parameter $\theta$ of one-parameter Gompertz life distribution is given as

$F(x ; \theta)=1-\exp \left(-\theta\left(e^{x}-1\right)\right) ; x>0, \theta>0$.

One of the main objectives of the life researchers is to reduce the cost and time of the experiments. To fulfill the desired objective, there are several types of censoring patterns available in the literature. One of the most common censoring patterns is Progressive Type-II censoring. On this criterion, a well-organized scheme has used for the removal of a pre-specified number of surviving test units at each failure time during the experiments. The elimination of test units before the failure may be planned to save the time and experimental cost. See [1] for more details regarding the Progressive censoring scheme. Several literatures are available on Progressive censoring, a little few of them are, [2], [3], [4], [5] and [6].

Johnson [7] design a life test in which the experimental units divided into a number of groups and all these groups are considered as the test units and run simultaneously. The life test will be terminated, when the first failure is observed in each group. This process is called the first failure censoring process. Wu \& Kus [8] combined the Progressive censoring scheme with first failure censoring, and named as First-Failure Progressive (FFP) censoring scheme. In this process, remove some test units from each group before first failures occurred. The Progressive Type-II censoring, First-Failure censoring, Type-II Censoring and complete sample case are the special cases of the FFP censoring and obtained by some minor modification. Based on all possible special cases of FFP censoring, under the CS-PALT, a comparative analysis has been presented in this study between different bound lengths with the help of simulated data.

\section{CS-PALT under FFP censoring}

The partially accelerated life testing (PALT) is widely applicable in such test situation in which, tough to collect lifetimes of highly reliable products with a long lifetime under normal test conditions. In PALT, some test units are kept under higher stress level and the rest is in normal test condition. In the present study, the concern life test criterion is CS-PALT, in which some of test units are kept in normal stress condition and some of test units are at a constant level of stress condition.

Suppose, from total of $n$ test units, $n_{1}$ test units selected randomly, are run at the normal test condition and remaining $n_{2}\left(=n-n_{1}\right)$ (say) test units are tested in accelerated test condition The probability density function, distribution function and failure rate of the Gompertz distribution are given under the normal test condition as

$$
f_{1}\left(x_{1} ; \theta\right)=\theta e^{x_{1}} \exp \left(-\theta\left(e^{x_{1}}-1\right)\right),
$$

$$
F_{1}\left(x_{1} ; \theta\right)=1-\exp \left(-\theta\left(e^{x_{1}}-1\right)\right)
$$


and

$$
\rho_{1}\left(x_{1}\right)=\theta e^{x_{1}} ; x_{1}>0, \theta>0 \text {. }
$$

Under accelerated test condition, if $\rho_{2}\left(x_{2}\right)$ is denoted by the failure rate function for an item and if $\beta(>1)$ be the acceleration factor, then the failure rate function under the stress condition is defined as

$$
\rho_{2}\left(x_{2}\right)=\beta \rho_{1}\left(x_{1}\right)=\beta \theta e^{x_{2}} ; x_{2}>0, \theta>0, \beta>1 .
$$

Using Eq. (5), the probability density function, and distribution function under the accelerated test condition are obtained as

$$
f_{2}\left(x_{2} ; \theta, \beta\right)=\theta \beta e^{x_{2}} \exp \left(-\theta \beta\left(e^{x_{2}}-1\right)\right)
$$

and

$$
F_{2}\left(x_{2} ; \theta, \beta\right)=1-\exp \left(-\theta \beta\left(e^{x_{2}}-1\right)\right) .
$$

Let us assume $n$ be the total test units under study. Under CS-PALT, total of $n$ test units divided into two test groups, $n_{1}$ test units are at normal test condition and $n_{2}$ test units at the accelerated test condition. Applying the FFP censoring on this situation, now each group further break into $k$ groups with an equal number of test units. Following, [9], the joint probability density function of order statistics based on the FFP censoring scheme under CS-PALT is defined as

$$
\begin{aligned}
L(\theta, \beta \mid \underline{x}) & \propto\left\{\prod_{i=1}^{m_{1}} f_{1}\left(x_{1 i} ; \theta\right)\left(1-F_{1}\left(x_{1 i} ; \theta\right)\right)^{k\left(R_{1 i}+1\right)-1}\right\} \\
& \times\left\{\prod_{i=1}^{m_{2}} f_{2}\left(x_{2 i} ; \theta, \beta\right)\left(1-F_{2}\left(x_{2 i} ; \theta, \beta\right)\right)^{k\left(R_{2 i}+1\right)-1}\right\} .
\end{aligned}
$$

Here, $R_{1 i}$ and $R_{2 i}$ are the progressive censoring scheme assumed for normal and accelerated test group respectively. Simplifying Eq. (8) as

$$
\begin{array}{r}
L(\theta, \beta \mid \underline{x}) \propto\left\{\prod_{i=1}^{m_{1}} \theta e^{x_{1 i}} \exp \left(-\theta\left(e^{x_{1 i}}-1\right)\right)\right. \\
\left.\times\left(\exp \left(-\theta\left(e^{x_{1 i}}-1\right)\right)\right)^{k\left(R_{1 i}+1\right)-1}\right\} \\
\times\left\{\prod_{i=1}^{m_{2}} \theta \beta e^{x_{2 i}} \exp \left(-\theta \beta\left(e^{x_{2 i}}-1\right)\right)\right. \\
\left.\times\left(\exp \left(-\theta \beta\left(e^{x_{2 i}}-1\right)\right)\right)^{k\left(R_{2 i}+1\right)-1}\right\}
\end{array}
$$$$
\Rightarrow L(\theta, \beta \mid \underline{x}) \propto \theta^{m_{1}+m_{2}} \beta^{m_{2}} \exp \left\{-\theta\left(T_{\left(x_{1}\right)}+\beta T_{\left(x_{2}\right)}\right)\right\}
$$

where $T_{\left(x_{1}\right)}=k \sum_{i=1}^{m_{1}}\left(1+R_{1 i}\right)\left(e^{x_{1 i}}-1\right) \quad$ and $\quad T_{\left(x_{2}\right)}=$ $k \sum_{i=1}^{m_{2}}\left(1+R_{2 i}\right)\left(e^{x_{2 i}}-1\right)$.

\section{ML estimation \& approximate confidence lengths}

Taking the logarithm of the joint distribution given in Eq. (9), as

$\log L(\theta, \beta \mid \underline{x})=\left(m_{1}+m_{2}\right) \log \theta+m_{2} \log \beta-\theta\left(T_{\left(x_{1}\right)}+\beta T_{\left(x_{2}\right)}\right)$.

The first and second order derivative of the log likelihood function given in Eq. (10), are obtained as

$$
\begin{aligned}
& \frac{\partial}{\partial \theta} \log L(\theta, \beta \mid \underline{x})=\frac{m_{1}+m_{2}}{\theta}-\left(T_{\left(x_{1}\right)}+\beta T_{\left(x_{2}\right)}\right) \\
& \frac{\partial^{2}}{\partial \theta^{2}} \log L(\theta, \beta \mid \underline{x})=-\frac{m_{1}+m_{2}}{\theta^{2}}
\end{aligned}
$$

$$
\frac{\partial}{\partial \beta} \log L(\theta, \beta \mid \underline{x})=\frac{m_{2}}{\beta}-\theta T_{\left(x_{2}\right)}
$$$$
\frac{\partial^{2}}{\partial \beta^{2}} \log L(\theta, \beta \mid \underline{x})=-\frac{m_{2}}{\beta^{2}}
$$

and

$$
\frac{\partial^{2}}{\partial \theta \partial \beta} \log L(\theta, \beta \mid \underline{x})=\frac{\partial^{2}}{\partial \beta \partial \theta} \log L(\theta, \beta \mid \underline{x})=T_{\left(x_{2}\right)}
$$

The Maximum Likelihood (ML) estimator corresponding to the parameter $\theta$ and $\beta$ are given as

$$
\hat{\theta}_{M L}=\frac{m_{1}+m_{2}}{T_{\left(x_{1}\right)}+\hat{\beta}_{M L} T_{\left(x_{2}\right)}}
$$

and

$$
\hat{\beta}_{M L}=\frac{m_{2}}{\hat{\theta}_{M L} T_{\left(x_{2}\right)}} .
$$

Both the expressions (Eq. 11-12) involved unknown parameter. A numerical technique (Newton Raphson integral method) is applied here for the numerical findings of these ML estimates.

The asymptotic variances and co-variances of the Maximum Likelihood estimators $\hat{\theta}_{M L}$ and $\hat{\beta}_{M L}$ of the parameters $\theta$ and $\beta$ are obtained by the elements of the inverse of the Fisher information matrix. The observed asymptotic variance-covariance matrix for the ML estimators is obtained as

$$
\begin{gathered}
{\left[\begin{array}{cc}
-\frac{\partial^{2}}{\partial \theta^{2}} \log L(\theta, \beta \mid \underline{x}) & -\frac{\partial^{2}}{\partial \theta \partial \beta} \log L(\theta, \beta \mid \underline{x}) \\
-\frac{\partial^{2}}{\partial \beta \partial \theta} \log L(\theta, \beta \mid \underline{x}) & -\frac{\partial^{2}}{\partial \beta^{2}} \log L(\theta, \beta \mid \underline{x})
\end{array}\right]_{\left(\hat{\theta}_{M L}, \hat{\beta}_{M L}\right)}^{-1}} \\
\quad=\left[\begin{array}{cc}
\operatorname{Var}\left(\hat{\theta}_{M L}\right) & \operatorname{Cov}\left(\hat{\theta}_{M L}, \hat{\beta}_{M L}\right) \\
\operatorname{Cov}\left(\hat{\beta}_{M L}, \hat{\theta}_{M L}\right) & \operatorname{Var}\left(\hat{\beta}_{M L}\right)
\end{array}\right]
\end{gathered}
$$

The unknown parameters $\theta$ and $\beta$ are involved in the expressions of the second derivative. Hence, replacing the parameters by their corresponding ML estimators for obtaining the Fisher information matrix. Thus, $(1-\tau) 100 \%$ Approximate Confidence Intervals (ACI) for the parameters $\theta$ and $\beta$ are obtained respectively as 


$$
\hat{\theta}_{M L} \mp Z_{\frac{\tau}{2}} \sqrt{\operatorname{Var}\left(\hat{\theta}_{M L}\right)}
$$

and

$$
\hat{\beta}_{M L} \mp Z_{\frac{\tau}{2}} \sqrt{\operatorname{Var}\left(\hat{\beta}_{M L}\right)} .
$$

Here, $Z_{\frac{\tau}{2}}$ is the percentile of the standard normal distribution with right-tail probability $\frac{\tau}{2}$. The applicability of the normal approximation in ML estimation is for small samples. Meeker [10], discussed a log-transformation, for improvements in the performance of the normal approximation. Hence, $(1-\tau) 100 \%$ improved approximate confidence intervals for the parameters under consideration are given as

$$
\left\{\hat{\theta}_{M L} \exp \left(\mp \frac{Z_{\frac{\tau}{2}} \sqrt{\hat{\theta}_{M L}}}{\hat{\theta}_{M L}}\right)\right\}
$$

and

$$
\left\{\hat{\beta}_{M L} \exp \left(\mp \frac{Z_{\tau} \sqrt{\hat{\beta}_{M L}}}{\hat{\beta}_{M L}}\right)\right\} \text {. }
$$

\section{Bootstrap confidence limits}

The bootstrap method is a re-sampling method for the confidence intervals ([11]). In the present section the confidence limits based on the parametric bootstrap method are obtained for the parameters under study.

The numerical values of ML Estimate of ML estimator $\hat{\theta}_{M L}$ and $\hat{\beta}_{M L}$ for the parameters $\theta$ and $\beta$ are obtained respectively from the Eq. (11) and Eq. (12), based on FFP censored data by using numerical techniques. Now, again generate two new independent samples of sizes $n_{1}$ and $n_{2}$ from the underlying distribution based on same censoring scheme.

Compute the bootstrap sample, by using ML estimators $\hat{\theta}_{M L}$ and $\hat{\beta}_{M L}$ and say $\hat{\hat{\theta}}_{M L}$ and $\hat{\hat{\beta}}_{M L}$, respectively from second generated samples by replacing ML estimates obtain from original progressive data. Repeat above step up to $N(=1,000)$ times to obtain $N$ different bootstrap samples. Arrange all these samples $\left(\hat{\hat{\theta}}_{M L}\right.$ and $\left.\hat{\hat{\beta}}_{M L}\right)$ in ascending order, for a final bootstrap sample of the form

$$
\zeta_{\theta}^{1} \leq \zeta_{\theta}^{2} \leq \ldots \leq \zeta_{\theta}^{N} \text { for } \hat{\hat{\theta}}_{M L}
$$

and

$$
\zeta_{\beta}^{1} \leq \zeta_{\beta}^{2} \leq \ldots \leq \zeta_{\beta}^{N} \text { for } \hat{\hat{\beta}}_{M L}
$$

Let us assume $g(x)=P\left(\zeta_{\Theta}^{*} \leq x\right)$ be the cumulative density function of $\zeta_{\Theta}^{*}(\forall \Theta=\theta, \beta$ be the final bootstrap samples). Then $(1-\tau) 100 \%$ approximate Percentile Bootstrap Confidence Limits for the parameters $\theta$ and $\beta$ are obtained respectively as

$$
\left[\zeta_{\theta(B)}^{*}\left(\frac{\tau}{2}\right), \zeta_{\theta(B)}^{*}\left(\frac{2-\tau}{2}\right)\right]
$$

and

$$
\left[\zeta_{\beta(B)}^{*}\left(\frac{\tau}{2}\right), \zeta_{\beta(B)}^{*}\left(\frac{2-\tau}{2}\right)\right] .
$$

\section{One-sample bayes prediction limits}

The one-sample Bayes prediction bound lengths have been investigated in the present section for the parameters under study. The one-parameter Gamma distribution is taken as the conjugate family of prior, for the scale parameter $\theta$ of the Gompertz distribution, and a vague prior is selected for the acceleration factor $\beta$, and are defined here as

$$
\pi_{\theta}=\frac{\theta^{\alpha-1} e^{-\theta}}{\Gamma(\alpha)} ; \alpha>0
$$

and

$$
\pi_{\beta}=\beta^{-1} ; \beta>0 .
$$

Under the Bayes theorem, the joint and marginal posterior densities corresponding to the parameters $\theta$ and $\beta$ are obtained and given as

$$
\begin{aligned}
& \pi_{(\theta, \beta)}^{*}=\Omega \theta^{m_{1}+m_{2}+\alpha-1} \beta^{m_{2}-1} \exp \left\{-\theta\left(T_{\left(x_{1}\right)}+\beta T_{\left(x_{2}\right)}\right)\right\} \\
& \pi_{(\theta)}^{*}=\Omega_{\theta} \theta^{m_{1}+\alpha-1} \exp \left\{-\theta\left(T_{\left(x_{1}\right)}+1\right)\right\}
\end{aligned}
$$

and

$$
\pi_{(\beta)}^{*}=\Omega_{\beta} \frac{\beta^{m_{2}-1}}{\left\{T_{\left(x_{1}\right)}+\beta T_{\left(x_{2}\right)}+1\right\}^{m_{1}+m_{2}+\alpha}}
$$

where $\Omega_{\theta}=\frac{\left(1+T_{\left(x_{1}\right)}\right)^{m_{1}+\alpha}}{\Gamma\left(m_{1}+\alpha\right)}, \Omega_{\beta}=\Omega \Gamma\left(m_{1}+m_{2}+\alpha\right)$ and $\Omega=\frac{1}{\Gamma\left(m_{2}\right)}$ $\frac{\left(T_{\left(x_{2}\right)}\right)^{m_{2}}\left(1+T_{\left(x_{1}\right)}\right)^{m_{1}+\alpha}}{\Gamma\left(m_{1}+\alpha\right)}$.

If we assume that, $\underline{x}\left(=x_{(1)}, x_{(2)}, \ldots, x_{\left(r_{1}\right)}\right)$ be the first $r_{1}$ ordered observed items from total test units and $\underline{y}\left(=y_{(1)}, y_{(2)}, \ldots, y_{\left(r_{2}\right)}\right)$ be the second independent ordered random sample of the future observations from the same model of size $r_{2}$. Then the Bayes predictive density function $h(y \mid \underline{x})$ (say) corresponding to the future random variable $Y$, is defined as

$$
h(y \mid \underline{x}) \propto \int_{\Theta} f(y ; \Theta) \pi^{*}(\Theta \mid \underline{x}) d \Theta .
$$

Solving Eq. (22) for the scale parameter $\theta$ and the acceleration parameter $\beta$, the Bayes predictive density functions are given respectively as

$$
h_{\theta}(y \mid \underline{x})=\Omega_{\theta} \Gamma\left(m_{1}+\alpha+1\right) \frac{e^{y}}{\left(T_{\left(x_{1}\right)}+e^{y}\right)^{m_{1}+\alpha+1}}
$$

and

$$
h_{\beta}(y \mid \underline{x})=\Omega_{\beta}^{*} \theta e^{y} e^{-\theta e^{y}} ;
$$

where $\Omega_{\beta}^{*}=\Omega_{\beta} e^{\theta} \int_{\beta} \frac{\beta^{m_{2}-1}}{\left\{T_{\left(x_{1}\right)}+\beta T_{\left(x_{2}\right)}+1\right\}^{m_{1}+m_{2}+\alpha}} d \beta$.

Following [12], the Bayes predictive one-sample bound length has obtained by solving following equality with coverage $(1-\tau)$,

$\operatorname{Pr}\left(l_{1} \leq Y \leq l_{2}\right)=1-\tau$.

Here, $l_{1}$ and $l_{2}$ be the lower and upper Bayes predictive bound limits respectively. Using Eq. (23) \& Eq. (25), the lower and upper Bayes 
predictive bound limits corresponding to the scale parameter $\theta$ are obtained as

$l_{\theta 1}=\log \left\{\left\{\left(1+T_{\left(x_{1}\right)}\right)^{-m_{1}-\alpha}-\frac{\tau}{2 \Omega_{\theta} \Gamma\left(m_{1}+\alpha\right)}\right\}^{-\frac{1}{m_{1}+\alpha}}-T_{\left(x_{1}\right)}\right\}$

and

$l_{\theta 2}=\log \left\{\left\{\left(1+T_{\left(x_{1}\right)}\right)^{-m_{1}-\alpha}-\frac{1-\tau}{2 \Omega_{\theta} \Gamma\left(m_{1}+\alpha\right)}\right\}^{-\frac{1}{m_{1}+\alpha}}-T_{\left(x_{1}\right)}\right\}$.

Similarly, the lower and upper Bayes predictive one-sample bound limits corresponding the parameter $\beta$ are obtained by using Eq. (24) \& Eq. (25) and, are given as

$$
l_{\beta 1}=\log \left\{-\frac{1}{\theta} \log \left(e^{-\theta}-\frac{\tau}{2 \Omega_{\beta}^{*}}\right)\right\}
$$

and

$$
l_{\beta 2}=\log \left\{-\frac{1}{\theta} \log \left(e^{-\theta}-\frac{1-\tau}{2 \Omega_{\beta}^{*}}\right)\right\} .
$$

The one-sample Bayes prediction bound lengths corresponding to the parameters $\theta$ and $\beta$ are given as

$$
L_{\theta}=l_{\theta 2}-l_{\theta 1}
$$

and

$$
L_{\beta}=l_{\beta 2}-l_{\beta 1} .
$$

\section{Numerical illustration}

A complete analysis has presented in this section by using simulated data. For simulation study, the value of the scale parameter $\theta$ has generated by using the prior distribution given in Eq. (18) with the help of pre assumed values of hyper-parameter $\alpha(=0.50,1.00,2.50)$. Using generated values of the scale parameter $\theta$, a set of 10,000 random samples has generated, each of size $n=30$ by using following relation

$$
x_{(i)}=\log \left\{1-\frac{1}{\theta} \log \left(1-U_{(i)}\right)\right\} .
$$

Here, the random variable $U_{(i)}$ is independently distributed uniform distribution with the parameter $(0,1)$. The Monte Carlo simulation technique was applied here for generating FFP censored samples for each simulation (See details for algorithms described in [13]). The Table (1), presents the different values of $k$ along with different special cases of the FFP censoring scheme. The values of censored sample sizes $m_{1}$ and $m_{2}$ are assumed equal, only for simplicity in the calculation.

The estimated values of maximum likelihood estimates $\hat{\theta}_{M L}$ and $\hat{\beta}_{M L}$ corresponding to the parameters $\theta$ and $\beta$ respectively, are given in Table (2) for the FFP censoring and their special cases. It observed from the table is that, as the hyper parameter $\alpha$ increases the magnitude of the ML estimate first increase for small $\alpha$ and then decreases for larger $\alpha$. Increasing trend also seen when the sample size getting larger. The maximum magnitude of the ML estimate is noted for the FFP censoring whereas the minimum is noted for the Type-II censoring for all the considered values. However, the magnitudes of ML estimates are smaller.

Maximum Likelihood approximate confidence lengths based on normal approximation have been obtained from the assumed values as discussed earlier and presented in the Table (3). The normal approximated ACL shows an increasing trend when confidence level increases and shows the maximum ACL for highest confidence value. Further, the sample size has increased the ACL increase. It is remarkable that, the maximum ACL noted for the FFP censoring scheme, whereas the complete sample case presents minimum ACL. It is further noted that, the magnitude of ACL without normal approximation is smaller when it compared ACL with normal approximation. However, the numerical findings are not presented here.

The Bootstrap confidence lengths and one-sample Bayes prediction bound lengths under FFP Censoring and their special cases are presented in the Table (4) - (5) respectively. All the properties have seen similar as discussed above. However, the one-sample Bayes prediction bound length shows a wider tendency as compared to Bootstrap and ACL for all considered parametric values. However, the bound lengths are robust.

\section{References}

[1] Balakrishnan, N. \& Aggarwala, R. 2000. Progressive Censoring Theory, Methods and Applications, Birkhauser Publishers, Boston.

[2] Ahmadia, J., Mir-Mostafaee, S. M. T. K. \& Balakrishnan, N. 2011 Bayesian prediction of k-record values based on progressively censored data from exponential distribution. Journal of Statistical Computation \& Simulation, 2011, 1 - 12

[3] Azimi, R., Yaghmaei, F. \& Azimi, D. 2012. Comparison of Bayesian estimation methods for Rayleigh Progressive censored data under the different asymmetric loss function. International Journal of Applied Mathematical Research, 1 (4), 452 - 461.

[4] Prakash, G. 2015. A comparative study based on Bayes estimation under different censoring criterion. Journal of Data Science, 13 (2), $261-280$

[5] Soliman, A. A., Abd-Ellah, A. H., Ahmed, E. A. \& Farghal, A. A 2015. Bayesian Estimation from Exponentiated Frechet Model using MCMC Approach based on Progressive Type-II Censoring Data Journal of Statistics Application and Probability, 4 (3), 387 - 403.

[6] Prakash, G. 2016. Some inference on progressive censored Gompertz data under random scheme. International Journal of Scientific Research, 6 (4), 290 - 299.

[7] Johnson, L. G. 1964. Theory and Technique of Variation Research Elsevier, Amsterdam, Netherlands.

[8] Wu, S. J. \& Kus, C. 2009. On estimation based on progressive first-failure censored sampling. Computational Statistics \& Data Analysis, 53, 3659 - 3670

[9] Prakash, G. 2017. First Failure Progressive Censored Weibull Data Under Bayesian Analysis. Statistics Research Letters, 6, 1 - 8.

[10] Meeker, W. Q. \& Escobar, L. A. 1998. Statistical methods for reliability data. John Wiley and Sons, New York.

[11] Kreiss, J. P. \& Paparoditis, E. 2011. Bootstrap methods for dependent data: a review. Journal of Korean Statistical Society, 40, 357 - 378.

[12] Prakash, G. \& Singh, D. C. 2013. Bayes prediction intervals for the Pareto model. Journal of Probability and Statistical Science, 11 (1), $109-122$.

[13] Balakrishnan, N. \& Sandhu, R. A. 1995. A simple simulation algorithm for generating Progressively Type-II censored samples. American Statistics, 49, $229-230$. 
Table 1: Special Cases of FFP Censoring Scheme

\begin{tabular}{c|l|l|l|l|l}
\hline Case & $k$ & $m_{1}$ & $m_{2}$ & $R_{i} ; 1,2, \ldots$, & Different Censoring Plans \\
\hline 1 & 5 & 05 & 05 & 12021 & First-Failure Progressive Type-II Censoring (FFP) \\
\hline 2 & 5 & 05 & 05 & 00000 & Progressive Type-II Censoring (PC) \\
\hline 3 & 1 & 05 & 05 & 12021 & First-Failure Censoring (FFC) \\
\hline 4 & 1 & 05 & 05 & 000025 & Type-II Censoring (T-II) \\
\hline 5 & 1 & 05 & 05 & 00000 & Complete Sample (CS) \\
\hline 1 & 5 & 10 & 10 & 1005001421 & First-Failure Progressive Type-II Censoring (FFP) \\
\hline 2 & 5 & 10 & 10 & 0000000000 & Progressive Type-II Censoring (PC) \\
\hline 3 & 1 & 10 & 10 & 1005001421 & First-Failure Censoring (FFC) \\
\hline 4 & 1 & 10 & 10 & 00000000020 & Type-II Censoring (T-II) \\
\hline 5 & 1 & 10 & 10 & 0000000000 & Complete Sample (CS) \\
\hline
\end{tabular}

Table 2: ML Estimate Under FFP Censoring Scheme

\begin{tabular}{|c|c|c|c|c|c|c|c|c|}
\hline & $m_{1}$ & $m_{2}$ & $\alpha$ & FFP & PC & FFC & T-II & $\mathrm{CS}$ \\
\hline \multirow{6}{*}{$\hat{\theta}_{M L}$} & \multirow{3}{*}{05} & \multirow{3}{*}{05} & 0.50 & 1.3444 & 1.3002 & 1.2380 & 1.0506 & 1.2467 \\
\hline & & & 1.00 & 1.3831 & 1.3375 & 1.2735 & 1.0908 & 1.2825 \\
\hline & & & 2.50 & 1.3719 & 1.3331 & 1.2494 & 1.0891 & 1.2786 \\
\hline & \multirow{3}{*}{10} & \multirow{3}{*}{10} & 0.50 & 1.3676 & 1.3068 & 1.2628 & 1.0824 & 1.2621 \\
\hline & & & 1.00 & 1.4069 & 1.3443 & 1.2990 & 1.1135 & 1.3083 \\
\hline & & & 2.50 & 1.3986 & 1.3398 & 1.2746 & 1.1022 & 1.2992 \\
\hline \multirow{6}{*}{$\hat{\beta}_{M L}$} & \multirow{3}{*}{05} & \multirow{3}{*}{05} & 0.50 & 1.1667 & 1.1384 & 1.0844 & 0.9118 & 1.0920 \\
\hline & & & 1.00 & 1.2102 & 1.1708 & 1.1152 & 0.9667 & 1.1213 \\
\hline & & & 2.50 & 1.1906 & 1.1569 & 1.0843 & 0.9452 & 1.1196 \\
\hline & \multirow{3}{*}{10} & \multirow{3}{*}{10} & 0.50 & 1.1969 & 1.1441 & 1.0959 & 0.9394 & 1.1053 \\
\hline & & & 1.00 & 1.2401 & 1.1967 & 1.1373 & 0.9864 & 1.1454 \\
\hline & & & 2.50 & 1.2138 & 1.1728 & 1.1162 & 0.9665 & 1.1305 \\
\hline
\end{tabular}


Table 3: Normal Approximated ACL Under FFP Censoring

\begin{tabular}{|c|c|c|c|c|c|c|c|c|c|}
\hline & $\tau$ & $m_{1}$ & $m_{2}$ & $\alpha$ & FFP & $\mathrm{PC}$ & FFC & T-II & $\mathrm{CS}$ \\
\hline \multirow{18}{*}{$\theta$} & \multirow{3}{*}{$90 \%$} & \multirow{3}{*}{5} & \multirow{3}{*}{5} & 0.50 & 1.1416 & 1.1034 & 1.0618 & 1.0417 & 0.9099 \\
\hline & & & & 1.00 & 1.1591 & 1.1290 & 1.0862 & 1.0684 & 0.9482 \\
\hline & & & & 2.50 & 1.1311 & 1.1120 & 1.0750 & 1.0576 & 0.9228 \\
\hline & \multirow{3}{*}{$95 \%$} & \multirow{3}{*}{5} & \multirow{3}{*}{5} & 0.50 & 1.2062 & 1.1393 & 1.1073 & 1.0697 & 1.0103 \\
\hline & & & & 1.00 & 1.2256 & 1.1413 & 1.1216 & 1.1024 & 1.0125 \\
\hline & & & & 2.50 & 1.2167 & 1.1404 & 1.1111 & 1.0925 & 1.0102 \\
\hline & \multirow{3}{*}{$99 \%$} & \multirow{3}{*}{5} & \multirow{3}{*}{5} & 0.50 & 1.2956 & 1.2362 & 1.1840 & 1.1447 & 1.0979 \\
\hline & & & & 1.00 & 1.3155 & 1.2690 & 1.1993 & 1.1917 & 1.1012 \\
\hline & & & & 2.50 & 1.3040 & 1.2406 & 1.1888 & 1.1682 & 1.0937 \\
\hline & \multirow{3}{*}{$90 \%$} & \multirow{3}{*}{10} & \multirow{3}{*}{10} & 0.50 & 1.1463 & 1.1140 & 1.0735 & 1.0584 & 0.9479 \\
\hline & & & & 1.00 & 1.1677 & 1.1319 & 1.0912 & 1.0753 & 0.9647 \\
\hline & & & & 2.50 & 1.1414 & 1.1238 & 1.0891 & 1.0685 & 0.9407 \\
\hline & \multirow{3}{*}{$95 \%$} & \multirow{3}{*}{10} & \multirow{3}{*}{10} & 0.50 & 1.2315 & 1.1756 & 1.1416 & 1.1137 & 1.0176 \\
\hline & & & & 1.00 & 1.2502 & 1.2065 & 1.1489 & 1.1436 & 1.0363 \\
\hline & & & & 2.50 & 1.2394 & 1.1798 & 1.1369 & 1.1362 & 1.0195 \\
\hline & \multirow{3}{*}{$99 \%$} & \multirow{3}{*}{10} & \multirow{3}{*}{10} & 0.50 & 1.3983 & 1.3590 & 1.2902 & 1.2702 & 1.1056 \\
\hline & & & & 1.00 & 1.4190 & 1.3718 & 1.3120 & 1.3077 & 1.1156 \\
\hline & & & & 2.50 & 1.4095 & 1.3635 & 1.2949 & 1.2745 & 1.1002 \\
\hline \multirow{18}{*}{$\beta$} & \multirow{3}{*}{$90 \%$} & \multirow{3}{*}{5} & \multirow{3}{*}{5} & 0.50 & 1.2443 & 1.2024 & 1.1567 & 1.1046 & 0.9898 \\
\hline & & & & 1.00 & 1.2636 & 1.2305 & 1.1735 & 1.1439 & 1.0318 \\
\hline & & & & 2.50 & 1.2328 & 1.2118 & 1.1712 & 1.1062 & 1.0039 \\
\hline & \multirow{3}{*}{$95 \%$} & \multirow{3}{*}{5} & \multirow{3}{*}{5} & 0.50 & 1.2530 & 1.2318 & 1.2167 & 1.1553 & 1.0901 \\
\hline & & & & 1.00 & 1.2666 & 1.2340 & 1.2324 & 1.1913 & 1.1025 \\
\hline & & & & 2.50 & 1.2369 & 1.2230 & 1.2208 & 1.1804 & 1.1010 \\
\hline & & & & 0.50 & 1.3936 & 1.3283 & 1.2709 & 1.2278 & 1.1763 \\
\hline & $99 \%$ & 5 & 5 & 1.00 & 1.4154 & 1.3643 & 1.2877 & 1.2794 & 1.1800 \\
\hline & & & & 2.50 & 1.4028 & 1.3331 & 1.2762 & 1.2536 & 1.1717 \\
\hline & & & & 0.50 & 1.2495 & 1.2140 & 1.1695 & 1.1129 & 1.0315 \\
\hline & $90 \%$ & 10 & 10 & 1.00 & 1.2730 & 1.2337 & 1.1890 & 1.1515 & 1.0540 \\
\hline & & & & 2.50 & 1.2541 & 1.2248 & 1.1867 & 1.1240 & 1.0336 \\
\hline & & & & 0.50 & 1.3531 & 1.2917 & 1.2543 & 1.2237 & 1.1181 \\
\hline & $95 \%$ & 10 & 10 & 1.00 & 1.3737 & 1.3257 & 1.2624 & 1.2565 & 1.1386 \\
\hline & & & & 2.50 & 1.3618 & 1.2963 & 1.2492 & 1.2484 & 1.1202 \\
\hline & & & & 0.50 & 1.5064 & 1.4632 & 1.3876 & 1.3656 & 1.1848 \\
\hline & $99 \%$ & 10 & 10 & 1.00 & 1.5291 & 1.4773 & 1.4116 & 1.4068 & 1.1958 \\
\hline & & & & 2.50 & 1.5187 & 1.4682 & 1.3928 & 1.3704 & 1.1789 \\
\hline
\end{tabular}


Table 4: Bootstrap Confidence Lengths Under FFP Censoring

\begin{tabular}{|c|c|c|c|c|c|c|c|c|c|}
\hline & $\tau$ & $m_{1}$ & $m_{2}$ & $\alpha$ & FFP & PC & FFC & T-II & $\mathrm{CS}$ \\
\hline \multirow{18}{*}{$\theta$} & \multirow{3}{*}{$90 \%$} & \multirow{3}{*}{5} & \multirow{3}{*}{5} & 0.50 & 1.0645 & 1.0476 & 1.0073 & 0.9879 & 0.8604 \\
\hline & & & & 1.00 & 1.1015 & 1.0752 & 1.0358 & 1.0204 & 0.9074 \\
\hline & & & & 2.50 & 1.0644 & 1.0459 & 1.0101 & 0.9933 & 0.8628 \\
\hline & \multirow{3}{*}{$95 \%$} & \multirow{3}{*}{5} & \multirow{3}{*}{5} & 0.50 & 1.1471 & 1.0823 & 1.0514 & 1.0150 & 0.9575 \\
\hline & & & & 1.00 & 1.1758 & 1.0943 & 1.0752 & 1.0566 & 0.9696 \\
\hline & & & & 2.50 & 1.1472 & 1.0734 & 1.0450 & 1.0270 & 0.9474 \\
\hline & \multirow{3}{*}{$99 \%$} & \multirow{3}{*}{5} & \multirow{3}{*}{5} & 0.50 & 1.2335 & 1.1761 & 1.1256 & 1.0875 & 1.0423 \\
\hline & & & & 1.00 & 1.2628 & 1.2178 & 1.1504 & 1.1430 & 1.0555 \\
\hline & & & & 2.50 & 1.2317 & 1.1703 & 1.1202 & 1.1003 & 1.0282 \\
\hline & \multirow{3}{*}{$90 \%$} & \multirow{3}{*}{10} & \multirow{3}{*}{10} & 0.50 & 1.0791 & 1.0478 & 1.0087 & 0.9940 & 0.8871 \\
\hline & & & & 1.00 & 1.1098 & 1.0824 & 1.0409 & 1.0237 & 0.9134 \\
\hline & & & & 2.50 & 1.0944 & 1.0773 & 1.0438 & 1.0238 & 0.9002 \\
\hline & \multirow{3}{*}{$95 \%$} & \multirow{3}{*}{10} & \multirow{3}{*}{10} & 0.50 & 1.1615 & 1.1074 & 1.0745 & 1.0476 & 0.9546 \\
\hline & & & & 1.00 & 1.1896 & 1.1473 & 1.0916 & 1.0865 & 0.9827 \\
\hline & & & & 2.50 & 1.1892 & 1.1315 & 1.0900 & 1.0864 & 0.9764 \\
\hline & \multirow{3}{*}{$99 \%$} & \multirow{3}{*}{10} & \multirow{3}{*}{10} & 0.50 & 1.3229 & 1.2849 & 1.2183 & 1.1990 & 1.0397 \\
\hline & & & & 1.00 & 1.3529 & 1.3073 & 1.2494 & 1.2453 & 1.0594 \\
\hline & & & & 2.50 & 1.3538 & 1.3002 & 1.2429 & 1.2231 & 1.0545 \\
\hline \multirow{18}{*}{$\beta$} & \multirow{3}{*}{$90 \%$} & \multirow{3}{*}{5} & \multirow{3}{*}{5} & 0.50 & 1.0436 & 1.0133 & 0.9744 & 0.9556 & 0.8322 \\
\hline & & & & 1.00 & 1.0736 & 1.0401 & 1.0020 & 0.9871 & 0.8780 \\
\hline & & & & 2.50 & 1.0293 & 1.0114 & 0.9768 & 0.9605 & 0.8342 \\
\hline & \multirow{3}{*}{$95 \%$} & \multirow{3}{*}{5} & \multirow{3}{*}{5} & 0.50 & 1.1097 & 1.0470 & 1.0170 & 0.9818 & 0.9262 \\
\hline & & & & 1.00 & 1.1378 & 1.0589 & 1.0404 & 1.0224 & 0.9382 \\
\hline & & & & 2.50 & 1.1095 & 1.0380 & 1.0106 & 0.9932 & 0.9161 \\
\hline & & & & 0.50 & 1.1934 & 1.1378 & 1.0889 & 1.0521 & 1.0082 \\
\hline & $99 \%$ & 5 & 5 & 1.00 & 1.2220 & 1.1785 & 1.1132 & 1.1061 & 1.0213 \\
\hline & & & & 2.50 & 1.1912 & 1.1319 & 1.0834 & 1.0641 & 0.9943 \\
\hline & & & & 0.50 & 1.0492 & 1.0134 & 0.9754 & 0.9612 & 0.8577 \\
\hline & $90 \%$ & 10 & 10 & 1.00 & 1.0755 & 1.0474 & 1.0073 & 0.9906 & 0.8835 \\
\hline & & & & 2.50 & 1.0590 & 1.0425 & 1.0021 & 0.9907 & 0.8710 \\
\hline & & & & 0.50 & 1.1233 & 1.0710 & 1.0392 & 1.0130 & 0.9230 \\
\hline & $95 \%$ & 10 & 10 & 1.00 & 1.1509 & 1.1099 & 1.0560 & 1.0510 & 0.9505 \\
\hline & & & & 2.50 & 1.1507 & 1.0949 & 1.0548 & 1.0541 & 0.9448 \\
\hline & & & & 0.50 & 1.2796 & 1.2428 & 1.1783 & 1.1596 & 1.0054 \\
\hline & $99 \%$ & 10 & 10 & 1.00 & 1.3090 & 1.2647 & 1.2087 & 1.2047 & 1.0248 \\
\hline & & & & 2.50 & 1.2901 & 1.2567 & 1.2027 & 1.1836 & 1.0204 \\
\hline
\end{tabular}


Table 5: One-Sample Bayes Prediction Bound Lengths Under FFP Censoring

\begin{tabular}{|c|c|c|c|c|c|c|c|c|c|}
\hline & $\tau$ & $m_{1}$ & $m_{2}$ & $\alpha$ & FFP & $\mathrm{PC}$ & FFC & T-II & $\mathrm{CS}$ \\
\hline \multirow{18}{*}{$\theta$} & \multirow{3}{*}{$90 \%$} & \multirow{3}{*}{5} & \multirow{3}{*}{5} & 0.50 & 1.1706 & 1.1360 & 1.0924 & 1.0713 & 0.9333 \\
\hline & & & & 1.00 & 1.2033 & 1.1658 & 1.1232 & 1.1065 & 0.9834 \\
\hline & & & & 2.50 & 1.1550 & 1.1350 & 1.0962 & 1.0780 & 0.9368 \\
\hline & \multirow{3}{*}{$95 \%$} & \multirow{3}{*}{5} & \multirow{3}{*}{5} & 0.50 & 1.2437 & 1.1736 & 1.1401 & 1.1007 & 1.0384 \\
\hline & & & & 1.00 & 1.2740 & 1.1857 & 1.1650 & 1.1449 & 1.0507 \\
\hline & & & & 2.50 & 1.2447 & 1.1647 & 1.1340 & 1.1146 & 1.0283 \\
\hline & \multirow{3}{*}{$99 \%$} & \multirow{3}{*}{5} & \multirow{3}{*}{5} & 0.50 & 1.3373 & 1.2751 & 1.2204 & 1.1792 & 1.1302 \\
\hline & & & & 1.00 & 1.3682 & 1.3195 & 1.2465 & 1.2385 & 1.1437 \\
\hline & & & & 2.50 & 1.3361 & 1.2697 & 1.2155 & 1.1939 & 1.1158 \\
\hline & \multirow{3}{*}{$90 \%$} & \multirow{3}{*}{10} & \multirow{3}{*}{10} & 0.50 & 1.1709 & 1.1371 & 1.0947 & 1.0788 & 0.9631 \\
\hline & & & & 1.00 & 1.2043 & 1.1728 & 1.1280 & 1.1093 & 0.9907 \\
\hline & & & & 2.50 & 1.1858 & 1.1674 & 1.1310 & 1.1094 & 0.9755 \\
\hline & \multirow{3}{*}{$95 \%$} & \multirow{3}{*}{10} & \multirow{3}{*}{10} & 0.50 & 1.2602 & 1.2016 & 1.1660 & 1.1368 & 1.0361 \\
\hline & & & & 1.00 & 1.2898 & 1.2440 & 1.1836 & 1.1781 & 1.0657 \\
\hline & & & & 2.50 & 1.2885 & 1.2260 & 1.1811 & 1.1803 & 1.0581 \\
\hline & \multirow{3}{*}{$99 \%$} & \multirow{3}{*}{10} & \multirow{3}{*}{10} & 0.50 & 1.4349 & 1.3938 & 1.3217 & 1.3007 & 1.1283 \\
\hline & & & & 1.00 & 1.4666 & 1.4172 & 1.3545 & 1.3500 & 1.1488 \\
\hline & & & & 2.50 & 1.4667 & 1.4185 & 1.3466 & 1.3252 & 1.1426 \\
\hline \multirow{18}{*}{$\beta$} & \multirow{3}{*}{$90 \%$} & \multirow{3}{*}{5} & \multirow{3}{*}{5} & 0.50 & 1.2636 & 1.2397 & 1.1918 & 1.1172 & 1.0170 \\
\hline & & & & 1.00 & 1.3038 & 1.2691 & 1.2094 & 1.1684 & 1.0710 \\
\hline & & & & 2.50 & 1.2615 & 1.2395 & 1.1970 & 1.1289 & 1.0217 \\
\hline & \multirow{3}{*}{$95 \%$} & \multirow{3}{*}{5} & \multirow{3}{*}{5} & 0.50 & 1.2927 & 1.2705 & 1.2547 & 1.1904 & 1.1220 \\
\hline & & & & 1.00 & 1.3170 & 1.2828 & 1.2811 & 1.2381 & 1.1450 \\
\hline & & & & 2.50 & 1.2658 & 1.2513 & 1.2490 & 1.2067 & 1.1235 \\
\hline & & & & 0.50 & 1.4400 & 1.3716 & 1.3115 & 1.2663 & 1.2124 \\
\hline & $99 \%$ & 5 & 5 & 1.00 & 1.4728 & 1.4193 & 1.3391 & 1.3304 & 1.2262 \\
\hline & & & & 2.50 & 1.4396 & 1.3666 & 1.3070 & 1.2833 & 1.1975 \\
\hline & & & & 0.50 & 1.2790 & 1.2419 & 1.1952 & 1.1359 & 1.0507 \\
\hline & $90 \%$ & 10 & 10 & 1.00 & 1.3137 & 1.2725 & 1.2257 & 1.1864 & 1.0842 \\
\hline & & & & 2.50 & 1.3039 & 1.2732 & 1.2333 & 1.1676 & 1.0729 \\
\hline & & & & 0.50 & 1.3876 & 1.3233 & 1.2841 & 1.2520 & 1.1414 \\
\hline & $95 \%$ & 10 & 10 & 1.00 & 1.4192 & 1.3689 & 1.3026 & 1.2964 & 1.1729 \\
\hline & & & & 2.50 & 1.4167 & 1.3481 & 1.2987 & 1.2979 & 1.1636 \\
\hline & & & & 0.50 & 1.5482 & 1.5029 & 1.4237 & 1.4007 & 1.2113 \\
\hline & $99 \%$ & 10 & 10 & 1.00 & 1.5820 & 1.5277 & 1.4589 & 1.4538 & 1.2328 \\
\hline & & & & 2.50 & 1.5811 & 1.5282 & 1.4492 & 1.4257 & 1.2251 \\
\hline
\end{tabular}

